\title{
ON RATIO SCALES OF WEIGHT
}

\author{
KAZUO NAKATANI
}

Sensyu Liniversity

\begin{abstract}
Stevens' power law is examined by magnitude estimation method and ratio rating method. Stimuli are nine plastic cylinders weighing from 40 to $200 \mathrm{~g}$. The criteria for prothetic-metathetic dichotomy are discussed in terms of ratio axioms and distribution functions. We find three kinds of biases, corresponding to the axioms, which originated from stimulus presentation procedures. Socalled ratio scale constructed by direct method cannot be conceived as a ratio scale in its original form, as it will not satisfy the axioms. After the biases are eliminated and the scale values are revised, we obtain power psychophysical equation. Psychological scaling methods are closely related to definitions about distribution function of sensation magnitude. Curvilinear relationship between category scale and ratio scale is an artifact due to the definitions.
\end{abstract}

Dichotomous classification of psychological continua into 'prothetic' and 'metathetic' ones was introduced by Stevens for traditional dichotomy between 'quantity ' and 'quality', such as loudness and pitch of tone (Stevens, 1940, 1951, p. 36). The classification presents one of the most important problems of psychological scaling, because its criteria are used as tools of testing the validity of the scaling procedures (Stevens, 1957). But its theoretical foundations remain unsolved. Luce and Galanter (1963, p. 302), basing on the aspect model, concluded that the metathetic continua include the prothetic ones as special cases of the former. While Eisler (1963), substituting a quantifiable characteristic 'protheticness' for the prothetic-metathetic dichotomy, has shown that metathetic continua could be regarded as a limiting case where increase in variability with magnitude drops to zero. In other words, the variability which is often named subjective jnd $J \Psi$ is constant over magnitude.

We should return to pragmatic criteria which might differentiate the continua. Original criteria mentioned by Stevens are

(1) the subjective size of $j$ nd,

(2) the form of category rating scale,

(3) the time order error and
(4) hysteresis.

Some of the existent scales are open to doubt about their validity through the discussion of the criteria. The four are not only concerned on scaling procedures, but also on all sorts of psychological measurements. It is probable, as Stevens has pointed out, that other criteria may be discovered if we continue to look for them. These criteria are not independent to each other. We will analyse them in accordance with the following basic concepts of scaling theories.

(A) Level of measurement-interval scale and ratio scale

Category scales on prothetic continua are known to show concave downward curves when plotted against ratio scales (Fig. 1). That is, a category scale $R$ satisfies approximately the logarithmic equation :

$$
R=a \log S+b
$$

and ratio scale $\Psi$ satisfies approximately the power function:

$$
\Psi=k S^{n}
$$

then,

$$
R=c \log \Psi+d
$$

where $S$ is physical value of stimulus and $c=a \mid n>0$. 
While on metathetic continua, category scales are said to be more or less linearly related to ratio scales.

Category scales are usually constructed by procedures which belong to the indirect methods, such as method of successive intervals and method of paired comparisons, and ratio scales are constructed by procedures which belong to direct methods, such as magnitude estimation method and ratio rating method (Stevens, 1957; Tanaka, 1961). As the difference assumption are usually adopted for the indirect methods, the category scales constructed are regarded to be at the level of interval scale (Gulliksen, 1961). And in the case of direct methods, we assume that the subjects' numerical reports represent the ratios of sensation magnitudes so that the constructed scales are deemed to belong to ratio scales.

If $R$ has the characteristics of interval scale, the subjective distance function $R\left(S_{i}, S_{j}\right)$ should satisfy the following distance axioms :

$$
\begin{array}{ll}
\text { i) } & R\left(S_{i}, S_{i}\right)=0 \\
\text { ii) } & R\left(S_{i}, S_{j}\right)=-R\left(S_{j}, S_{i}\right) \\
\text { iii) } & R\left(S_{i}, S_{j}\right)+R\left(S_{j}, S_{k}\right) \geqslant R\left(S_{i}, S_{k}\right) .
\end{array}
$$

For example, when Fechnerian law (1) is verified by experiment on absolute judgements, the equation

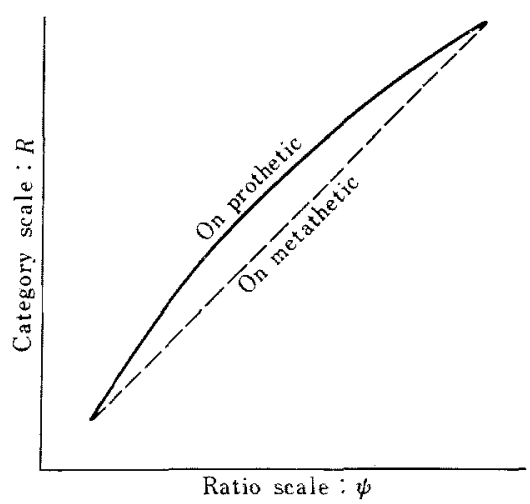

FIG. 1. Relation between two scales.

$$
\begin{aligned}
R\left(S_{i}, S_{j}\right) & =\left(a \log S_{i}+b\right)-\left(a \log S_{j}+b\right) \\
& =a \log \left(S_{i} / S_{j}\right)
\end{aligned}
$$

should be confirmed by experiment on pair comparisons. And if $\Psi$ is at the level of ratio scale, the subjective ratio function $\Psi\left(S_{i}, S_{j}\right)$ should satisfy following ratio axioms :

$$
\begin{array}{ll}
\text { i) } & \Psi\left(S_{i}, S_{i}\right)=1 \\
\text { ii) } & \Psi\left(S_{i}, S_{j}\right)=1 / \Psi\left(S_{j}, S_{i}\right) \\
\text { iii) } & \Psi\left(S_{i}, S_{j}\right) \cdot \Psi\left(S_{j}, S_{k}\right)=\Psi\left(S_{i}, S_{k}\right) .
\end{array}
$$

For example, when Stevens' law is obtained by magnitude estimations, the equation

$$
\begin{aligned}
\Psi\left(S_{i}, S_{j}\right) & =\left(k S_{i}^{n}\right) /\left(k S_{j}^{n}\right) \\
& =\left(S_{i} / S_{j}\right)^{n}
\end{aligned}
$$

should be proved by ratio rating experiment (Nakatani, 1965). Most of the subjective differences which have been scaled by pair comparison methods do not satisfy the distance axioms and show systematic deviations from those expected from the axioms (Tanaka \& Nakatani, 1967). If we want true interval scale which will satisfy the distance axioms, we should eliminate the possible biases to revise the original scale. Tanaka and Nakatani, eliminating the biases, found the revised category scale $R^{\prime}$ fits a linear psychophysical equation:

$$
R^{\prime}=a^{\prime} S+b^{\prime} .
$$

There remains the same type of problem whether $\Psi$ satisfies the ratio axioms or not. If the obtained $\Psi$ shows some deviations from the axioms, we must eliminate the possible biases and find the psychophysical function for the revised $\Psi^{\prime}$.

(B) Indirect and direct method

The model for indirect methods postulates usually a psychological continuum upon which the values of the discriminal processes distribute normally. Thurstonian equation of comparative judgement 


$$
\begin{aligned}
R_{i}-R_{j} & =Z_{i, j}\left\{\operatorname{Var}\left(R_{i}\right)+\operatorname{Var}\left(R_{j}\right)\right. \\
& \left.-2 \operatorname{Cov}\left(R_{i,}, R_{j}\right)\right\}^{1 / 2}
\end{aligned}
$$

is a typical example of the application of the model for indirect methods. The case $\mathrm{V}$ solution which are used most frequently for its convenience of calculation assumes that

and

$$
\operatorname{Var}(R)=\text { constant }
$$

$$
\operatorname{Cov}\left(R_{i}, R_{j}\right)=\text { constant. }
$$

Direct methods are thought to be based on the subjects' direct assessments of sensation magnitudes without any postulates of distribution functions. But it is well known that the rated values about the magnitudes along prothetic continua show approximately logarithmic normal distributions and their standard deviations increase as the scale value increases. Goude (1962, p. 44) and others suggest that

$$
\operatorname{Var}^{1 / 2}(\Psi)=k \Psi
$$

Because of the logarithmic normal distribution of the direct estimations, $\log$ arithmic transformations of them should satisfy the following Thurstonian equation

$$
\begin{aligned}
\log \Psi_{i} & -\log \Psi_{j} \\
& =Z_{i j}\left\{\operatorname{Var}\left(\log \Psi_{i}\right)+\operatorname{Var}\left(\log \Psi_{j}\right)\right. \\
& \left.-2 \operatorname{Cov}\left(\log \Psi_{i}, \log \Psi_{j}\right)\right\}^{1 / 2} .
\end{aligned}
$$

We should better substitute the equation (9) by

$$
\operatorname{Var}\left(\log W^{\prime}\right)=\text { constant }
$$

and name it Case VI.

It is clear that equation (3) is conserved in the relation between the equation (7) and (10). Curvilinear relationship between category scale and ratio scale could be attributable to the fact that indirect methods are based on normal distribution assumption and direct methods on the subjects' numerical reports of logarithmic normal distributions. If that is the case, phenomenon presented in Fig. 1 should be conceived as an artifact caused by scaling procedures.

(C) Subjective size of jnd

The jnd on prothetic continua is said to increase its subjective size as psychological magnitude increases and it remains constant on metathetic ones (Stevens, 1957).

If we want to use the concept 'subjective size of $j n d$ ' in order to examine the validity of psychological scales, we must give it a clear definition. It may be reasonable that, just as physical size of jnd is represented by the difference between two stimuli on physical scale, its subjective size is defined by the difference between the psychological scale values corresponding to the stimuli. We can not determine it uniquely under the above definition, as far as we have more than two psychological scales on a modality. That is, $A R$ on category scale and $\Delta \Psi$ on ratio scale given by the following equations

$$
\begin{aligned}
& \Delta R=R(S+\Delta S)-R(S) \\
& \Delta \Psi=\Psi(S+\Delta S)-\Psi(S)
\end{aligned}
$$

equally deserve the name of subjective size of $\mathrm{jnd}$.

Then

$$
\begin{aligned}
J R & =\{a \log (S+J S)+b\}-\{a \log S+b\} \\
& =a \log (1+J S / S)
\end{aligned}
$$

and

$$
\begin{aligned}
\Delta W / \Psi & =\left\{k(S+\Delta S)^{n}-k S^{n}\right\} / k S^{n} \\
& =n(\Delta S / S)+n(n-1) / 2(\Delta S / S)^{2}+\ldots
\end{aligned}
$$

If Weber's law holds, i.e., $\Delta S / S=$ constant, we can rewrite (14) and (15) as follow

$$
\begin{aligned}
\Delta R & =\text { constant } \\
\Delta \Psi / W & =\text { constant }
\end{aligned}
$$

$\Delta R$ is proportional to discriminal dispersion on psychological continuum and $S T$ is proportional to standard deviation of sensation magnitude. Therefore the equation (16) and (17) are equivalent to (8) and (9) 
TABLE 1

List of scaling methods

\begin{tabular}{l|l|l}
\hline $\begin{array}{l}\text { Glass } \\
\text { Scale }\end{array}$ & $\begin{array}{l}\text { Indirect method } \\
\text { Category scale } \\
\text { Interval scale }\end{array}$ & $\begin{array}{l}\text { Direct method } \\
\text { Ratio scale } \\
\text { Ratio scale }\end{array}$ \\
\hline Rating procedure & $\begin{array}{l}\text { Category rating method } \\
\text { Successive interval method } \\
\text { Method of comparative judgement } \\
\text { Method of equal appearing interval }\end{array}$ & $\begin{array}{l}\text { Magnitude estimation method } \\
\text { Ratio rating method } \\
\text { Constant sum method }\end{array}$ \\
\hline \multirow{2}{*}{$\begin{array}{l}\text { Adjusting procedure } \\
\text { Equisection method }\end{array}$} & $\begin{array}{l}\text { Fractionation method } \\
\text { Multiplication method }\end{array}$
\end{tabular}

mentioned in (B). Subjective jnd on logarithmic interval scale can be calculated, by application of logarithmic transformation to equation (3), with the logarithmic increment :

$$
\begin{aligned}
\Delta \log \Psi & =\{n \log (S+\Delta S)+\log k\} \\
& -\{n \log S+\log k\} \\
& =n \log (1+\Delta S / S) \\
& =\text { constant }
\end{aligned}
$$

which is equivalent to (11) in (B). Discussions in (B) and (C) concerning to aspects of the criteria, such as scaling method, scale function, subjective jnd, and variability of sensation, are all reducible to the problem about the definition for distribution function of sensations.

Stevens based his theory only on the latter $J \Psi$ which is given by his power law and gave no account for the subjective size of $\Delta R$. The fact that jnd on metathetic continuum has turned out to be approximatelly equal in subjective size even when measured by magnitude scale, is probably due to the fact that we can not obtain the power function for the metathetic continuum.

(D) Constant error

It is an excellent principle of psychology since Fechner that fluctuations of obtained values are analyzed into some components which are devided into two broad classes: accidental errors and constant errors. Distribution functions and var- iances mentioned in $(\mathrm{B})$ and $(\mathrm{C})$ concern only with the former. The latter are characterizable by routines of stimulus presentations. For instance, time order error is based on the order of stimulus presentation, space order error is on the spacial arrangement of stimuli, hysteresis is on the direction of stimulus change such as ascending and discending series, and stimulus coupling effect is over- or underestimation due to the combination of the paired. According to Stevens, time order error and hysteresis are found on prothetic continua but not on metathetic ones, and so they have been used as the criteria which differentiate the two kinds of continua.

Time order error is said to be exhibited by comparative judgment and hysteresis by bisection and equisection. Comparative judgment is a typical example of categorical ones and bisection and equisection are categorical operations for stimulus adjustment. They lead to interval scalings. We can classify scaling methods as Table 1.

Constant errors have been detected by categorical methods listed in the left half of the table and said not to be found by ratio methods which are classified in the right half.

Subjective distances are likely to be over- or under-estimated in experiments of category methods. We can identify constant errors with three kinds of deviations from the distance axioms (Tanaka \& Naka- 
TABLE 2

Statistics of estimations

\begin{tabular}{l|ccccccccc}
\hline \multicolumn{1}{c|}{$\mathrm{S}$} & 40 & 60 & 80 & 100 & 120 & 140 & 160 & 180 & 200 \\
\hline $\mathrm{E}(\log \Psi)$ & 1.433 & 1.699 & 1.883 & 2.001 & 2.138 & 2.244 & 2.330 & 2.401 & 2.443 \\
$\Psi$ & 27.1 & 50.0 & 76.4 & 100.2 & 137.4 & 175.4 & 213.8 & 251.8 & 277.3 \\
$\operatorname{Var}(\log \Psi)$ & .017 & .021 & .012 & .003 & .010 & .013 & .017 & .015 & .015 \\
Skew $(\log \Psi)$ & .15 & -.74 & -1.58 & -.10 & .50 & .53 & .62 & .41 & -.03 \\
Kurt $(\log \Psi)$ & -.69 & .11 & 4.69 & 6.18 & .06 & .00 & .43 & .23 & -.78 \\
$\mathrm{E}(\Psi)$ & 28.3 & 52.7 & 78.6 & 101.1 & 141.3 & 181.9 & 223.9 & 263.5 & 288.6 \\
Var $(\Psi)$ & 73.4 & 249.7 & 287.3 & 186.2 & 1203.4 & 2685.2 & 5378.7 & 7396.5 & 6602.3 \\
Skew $(\Psi)$ & .91 & -.06 & .12 & 1.29 & 1.17 & 1.32 & 1.65 & 1.81 & .52 \\
Kurt $(\Psi)$ & 1.12 & -.62 & 3.83 & 5.40 & 1.87 & 2.26 & 4.28 & 7.89 & -.28
\end{tabular}

tani, 1967). Inequality which denies the axiom (i)

$$
\text { i*) } R\left(S_{i}, S_{i}\right) \neq 0
$$

is no more than mathematical expression of time order error, provided that the order of the two $S_{i}$ in the parentheses coincides with the order of stimulus presentations. So is the case with hysteresis. Inequality contradicting to the axiom (ii)

$$
\text { ii*) } R\left(S_{i}, S_{j}\right) \neq-R\left(S_{j}, S_{i}\right)
$$

means that subjective interval estimated for stimulus difference in ascending order (for example, from $S_{i}$ to $S_{j}$, if $S_{i}<S_{j}$ ) does not appear equal to that of the difference in discending order (from $S_{j}$ to $S_{i}$ ). And coupling effect is the phenomenon that, when mediated by third stimulus $S_{j}$, the distance between $S_{i}$ and $S_{k}$ can not be predicted by the sum of the distance between $S_{i}$ and $S_{j}$ and that of $S_{j}$ and $S_{k}$, even if the three stimuli are in linear array. This is equivalent to the negation of the axiom (iii) ;

$$
\text { iii*) } R\left(S_{i}, S_{j}\right)+R\left(S_{j}, S_{k}\right) \neq R\left(S_{i}, S_{k}\right) .
$$

Few discussions have been done about what will distort the ratio rating and ratio adjustment. Is it true that we can not find the biases by ratio methods, which might correspond to the above mentioned constant errors, even on prothetic continua?
This can be clarified in the process of examining the deviations from the ratio axioms.

\section{EXPERIMENT I}

We have discussed that the criteria are closely related to each other. They are eventually attributable to two problems. The one is about distribution functions and the other is about the distance and ratio axioms. The purpose of the present experiment is to construct the ratio scale about subjective heaviness and to examine about distributions of the judgments.

\section{Method}

Magnitude estimation method with prescribed modulus was used as a typical example of direct method. Stimuli used were made of green plastic cylinders $(30 \mathrm{~mm}$ diameter, $50 \mathrm{~mm}$ height), flled with plumbum shots. Standard stimulus was $100 \mathrm{~g}$, and variable stimuli were $40,60,80,100,120,140,160,180$ and $200 \mathrm{~g}$. Subjects were requested to assign 100 to apparent heaviness of the standard and to estimate the magnitude of variable stimulus which, always paired with the standard, were presented randomly for 22 trials. (First 2 trials were discarded as warming-up). The subjects were 8 undergraduate students of University of Tokyo.

Results

(1) Magnitude scale values $\psi$, calculated by logarithmic means, are shown in 


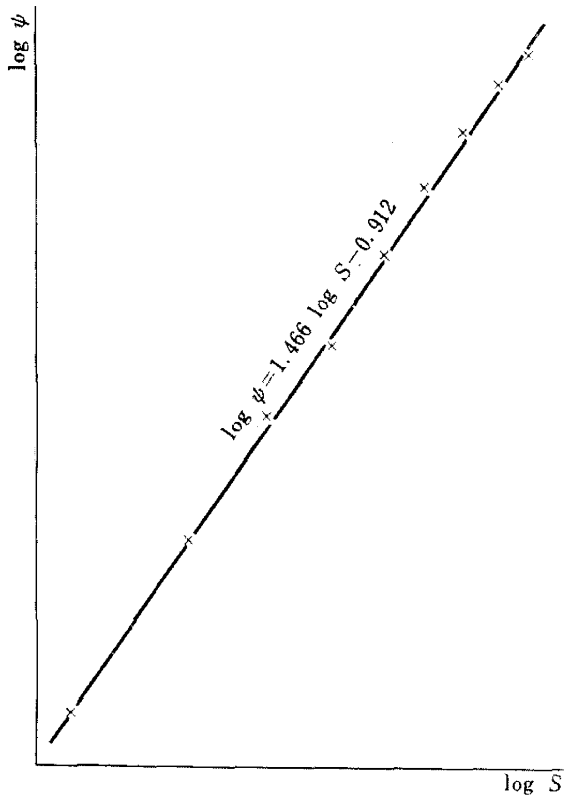

FIG. 2. Linear relation between $\log S$ and $\log \Psi$.

the second raw of Table 2. The relation between $\log \Psi$ and $\log S$ is linear (Fig. 2). Application of the method of least squares has proved the regression function to be

$$
\log \Psi=1.466 \log S-0.912 .
$$

That is, our psychophysical equation is

$$
\Psi=0.122 S^{1.466} .
$$

Therefore, Stevens' power law seems to add another supporting evidence, with characteristic exponent 1.466 , a nearest value to 1.45 suggested by Stevens. As the coefficient is arbitrary, we can chose the subjective value of $100 \mathrm{~g}$ as unit and transform the equation (20) into the form of veg scale

$$
\mathrm{veg}_{\mathrm{Exp} . \mathrm{I}}=0.00122 S^{\mathbf{1 . 4 6 6}} .
$$

(2) Standard deviation of estimated magnitudes increases as stimulus increases, though the proportional equation (9) can not be seen as shown in Fig. 3.

It seems that logarithmic variances (third raw of Table 2) approximately fit the equation (11), except the cave-in at the

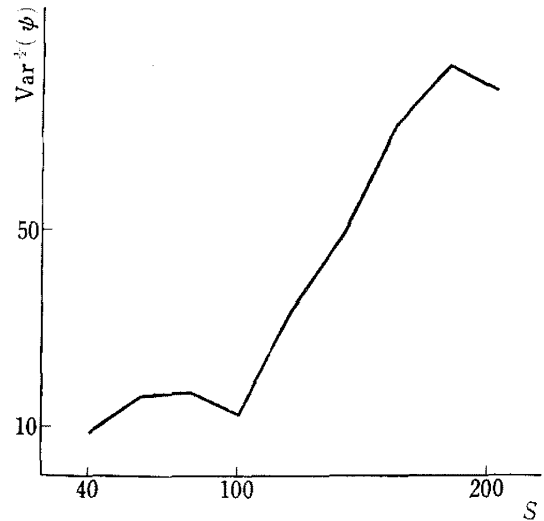

FIG. 3. Standard deviation of estimated values.

$100 \mathrm{~g}$. When the variable stimulus is near the standard stimulus, the estimation seems to be fairly restrained of the variability. We can explain this phenomenon by the increase of covariance term in the equation (10). It may be probable that when two stimuli $S_{i}$ and $S_{j}$ are similar in pair comparison, correlation $r_{i j}$ and so covariance $\operatorname{Cov}(i, j)=r_{i j} \operatorname{Var}^{1 / 2}(i) \operatorname{Var}^{1 / 2}(j)$ on psychological continuum becomes larger and that the appearing variance becomes smaller.

(3) Skewness and kurtosis are calculated about the estimated magnitudes and their logarithmically transformed values, by which we can compare fitness of the normal and of the logarithmic normal distribution functions (Table 2). The skewness of magnitude (8th raw of Table 2) shows that their distributions are positively skewed, and after the logarithmic transformation the skewness values fluctuate around zero (4th raw). The kurtosis (9th raw) shows that the distributions are more flat than the normal, those of logarithms (5th raw) are very near to zero except the cases of the 80 and $100 \mathrm{~g}$. The high kurtosis of these two are due to the small variances mentioned in section (2). We may conclude that distribution function of estimated magnitude is logarithmic normal rather than normal.

(4) The fact that distributions of direct estimations of sensation magnitudes are 
TABLE 3

$\log \Psi_{i .}$ and $\log \Psi_{. i}$

\begin{tabular}{c|ccccccccc}
\hline $\mathrm{S}$ & 40 & 60 & 80 & 100 & 120 & 140 & 160 & 180 & 200 \\
\hline $\log \Psi_{i .}$ & -.689 & -.441 & -.226 & -.052 & .060 & .163 & .254 & .339 & .405 \\
$\log \Psi_{. i}$ & -.475 & -.343 & -.183 & -.051 & .051 & .157 & .285 & .341 & .404 \\
$\log \widehat{\Psi}_{i}$ & -.582 & -.392 & -.205 & -.052 & .056 & .160 & .270 & .340 & .405
\end{tabular}

logarithmic normal should not be taken to reject Thurstonian assumption that discriminal processes underlying category ratings fluctuate by normal distribution functions. One of the most important purposes of psychological scaling may be in the statistical prediction of sensation. Therefore it is reasonable to define the sensation by such $R$ as in equation (7) which will distribute normally. Likewise we may take $\Psi=\log \Psi$, instead of $\Psi$ itself, as the psychological scale value whose distribution is the normal. In this case the Fechnerian psychophysical equation will be available;

$$
\Psi=n \log S+\log k .
$$

The curvilinear relation between $R$ and $\Psi$ is reducible to the problem of researcher's taste about the definition of distribution function. However, the coefficient $n$ of the above $(1 *)$ is invariant which is characteristic to modarity, while the corresponding coefficient $a$ of Fechnerian equation (1) is arbitrary. We are left to study whether we can find any invariant quantity by categorical methods which lead to logarithmic law, and why the exponent $n$ is obtained to be invariant by ratio methods which lead to power law. These problems will be examined in another study.

\section{EXPERIMENT II}

We will examine the second problem, that is, whether scaling of ratio judgments is possible at the level of ratio scale. If we find any systematic deviations from the ratio axioms, we should eliminate them and revise the scale values so as to satisfy the axioms. Psychophysical equation will be calculated between the revised psychological scale and physical scale.

Method

The stimuli were of the same type as used in Exp. I, weighing 40,60, 80,100,120,140, 160,180 and $200 \mathrm{~g}$. All of the possible pairs (81 pairs) were used, and each pair was presented 5 times at random. Subjects were requested to report subjective ratios of paired sensation magnitudes in the form of percentiles. Six students majoring in psychology served as subjects.

Results

We obtain logarithmic ratio matrix || $\log \Psi_{i j} \|$, by logarithmic means minus 2.00 of the estimated percentiles, where suffixes $i$ and $j$ denote stimulus pair $\left(S_{i}, S_{j}\right)$.

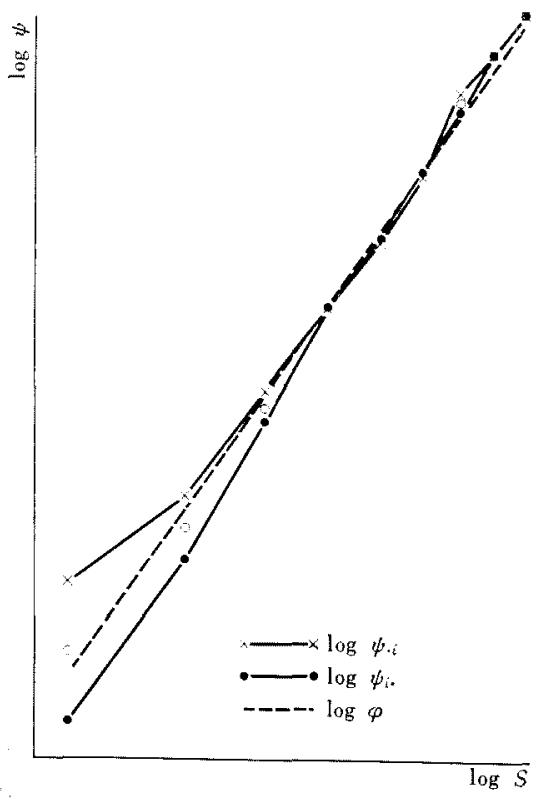

FrG. 4. $\log \Psi_{i \text {. }}$ and $\log \Psi_{. i}$ are plotted against $\log S$. They show deviations from linearity. 


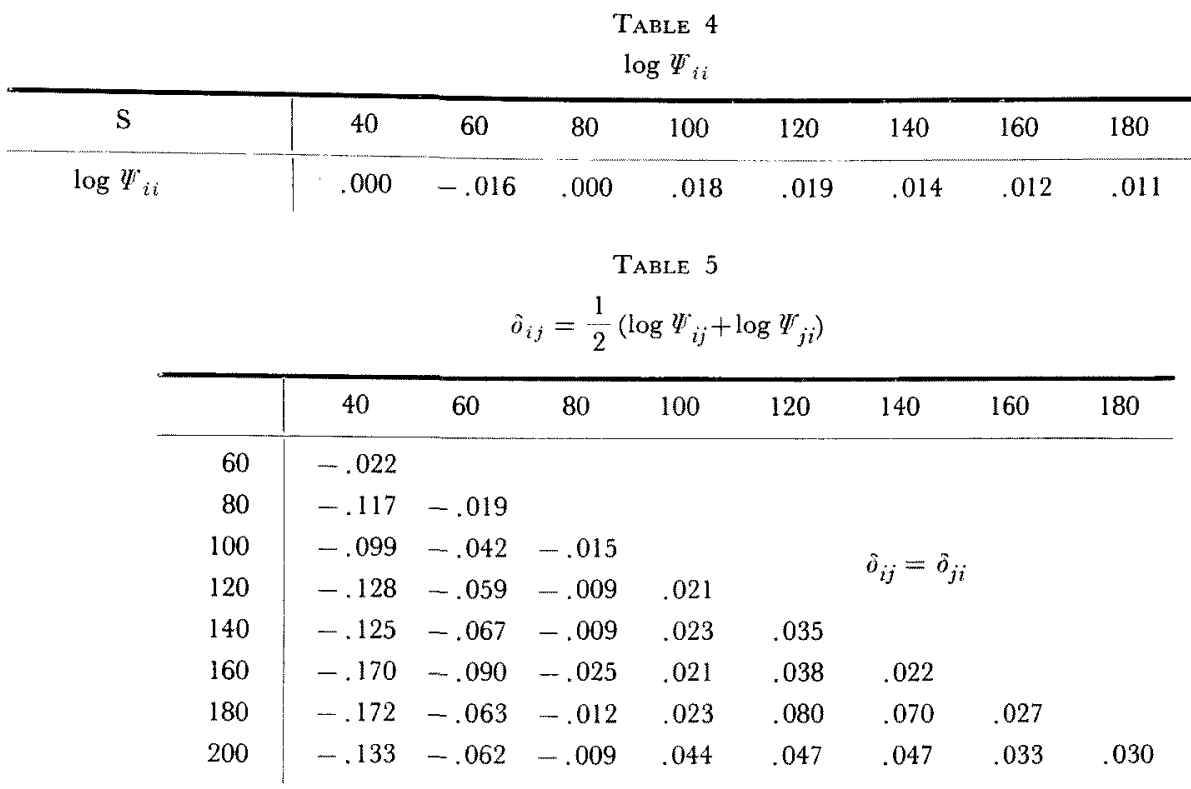

If $\log \Psi_{i j}$ does not contain any constant error components,

$$
\log \Psi_{i j}=\log \Psi_{j}-\log \Psi_{i}+e_{i, j}
$$

where $\Psi_{i}$ and $\Psi_{j}$ are psychological scale values of stimulus $i$ and $j$, and $e_{i j}$ means accidental error.

When the arbitrary unit of measurement is chosen so that

$$
\sum_{i} \log \Psi_{i}=0
$$

we can get estimates of $-\log \Psi_{i}$ and $\log \Psi_{j}$ by raw mean and column mean as follows

$$
\begin{aligned}
-\log \Psi_{i .} & =1 / m \sum_{k} \log \Psi_{i k} \\
\log \Psi_{, j} & =1 / m \sum_{k} \log \Psi_{k j}
\end{aligned}
$$

where $m=9$, the number of stimuli.

Equation (24) and (25) will yield two sets of scale values for the same stimuli. These sets will not be identical because of error component. They may be averaged;

$$
\log \hat{\Psi}_{i}=\left(\log \Psi_{i .}+\log \Psi_{. i}\right) / 2
$$

When the error component is no more than accidental, fluctuations of $\log \Psi_{i}$. and $\log \Psi_{. i}$ around the average (26) will be negligibly small.
Experimentally obtained values are shown in Table 3 which are plotted against $\log S$ in Fig. 4.

The average scale values $\log \hat{T}$ coincide with a straight line in the Jog-log coordinate. Application of the method of least squares leads to the following equation

$$
\log \hat{\Psi}=1.452 \log S-2.948 \text {. }
$$

As -2.948 can be altered arbitrarily according to the choice of modulus, we take I veg as subjective modulus for $S=100 \mathrm{~g}$. Then

$$
v^{\text {veg }} g_{\text {Exp.II }}=0.00125 S^{1.452} \text {. }
$$

This is much agreeable to the so-called veg scale.

The raw mean scale $\log \Psi_{i}$, and the column mean scale $\log \Psi_{. i}$ show systematic deviations from the line, which are larger when stimuli are smaller. We can modify power equations for the two scales by

$$
\Psi=k\left(S \pm S_{0}\right)^{n}
$$

as Stevens did for second order approximation of the power law. Of course the term $S_{0}$ in the equation (29) does not necessarily mean the effective threshold parameter. 
TABLe 6

\begin{tabular}{r|rrrrrrrr}
\multicolumn{8}{c}{$\gamma_{i j}=\log \Psi_{i j}-\left(\log \Psi_{j}^{\prime}+\log \Psi_{i}^{\prime}\right)$} \\
\hline & \multicolumn{1}{c|}{40} & \multicolumn{1}{c}{60} & \multicolumn{1}{c}{80} & 100 & 120 & 140 & 160 & 180 \\
\hline 60 & .002 & & & & & & & \\
80 & .019 & .009 & & & & & & \\
100 & .003 & .037 & .044 & & & & & \\
120 & .010 & .004 & -.001 & .031 & & $\gamma_{i j}=-\gamma_{j i}$ & \\
140 & .002 & .019 & .020 & .025 & .005 & & & \\
160 & -.019 & -.035 & .000 & .025 & .021 & .045 & & \\
180 & -.017 & -.021 & -.018 & .003 & .020 & .019 & .017 & \\
200 & -.001 & -.012 & -.018 & .001 & -.002 & .008 & .020 & .004
\end{tabular}

TABLE 7

$\left\|\log \Psi_{i j}^{*}\right\|$

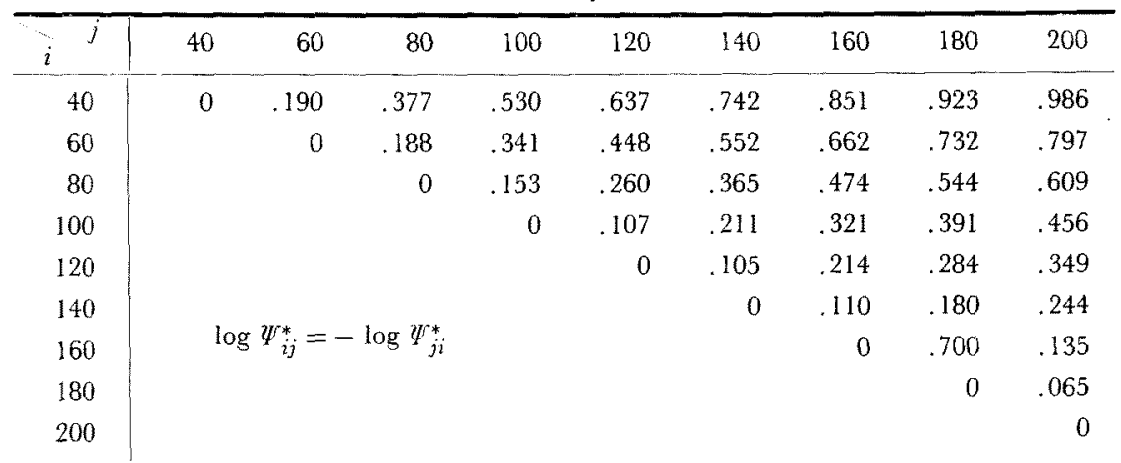

The deviations from linearity of our data is due to the effect of stimulus presentation order, that is, whether stimulus $i$ was presented at first or latter in the pair. We cannot neglect constant error components and have to revise the equation (22), so as to correspond to the case of category rating method (Tanaka \& Nakatani, 1967).

(2) Even when physical weights of paired stimuli are equal, the stimulus presented in the latter will usually be perceived slightly heavier. This is time order error. Six out of nine diagonal elements $\log \Psi_{i i}$ are positive (Table 4).

Average of them is

$$
1 / 9 \sum_{i} \log \Psi_{i i}=0.007=\log 1.016 .
$$

That is, estimation of the latter appears to be heavier 1.016 times. This is the same tendency as that of category methods.
The bias due to order of stimulus presentation indicates that the ratio axiom (i) does not hold unless we make some modification.

(3) It is possible that the bias due to the presentation order may also be found when the paired stimuli are not equal. We can express the bias by $\delta_{i j}$ which can be written as

$$
\delta_{i j}=1 / 2\left(\log \Psi_{i j}+\log \Psi_{j i}\right) .
$$

If the amount of $\delta$ is negligible, we can say that the ratio axiom (ii) is satisfied.

Table 5 shows that the obtained values of $\delta$ depend on the differences between the paired stimuli, which can not be ignored. Systematic tendency of this $\delta$ 's variability, however, does not coincide with that of $\delta$ obtained for categorical pair comparisons (Tanaka \& Nakatani, 1967). The values of $\delta$ are likely to be affected not only by the 
stimulus presentation order, but also by styles of judgments, stimulus range, number, step intervals and so on. that

(4) Let's define $\log \Psi_{i j}^{\prime}$ in such a way

$$
\begin{gathered}
\log \Psi_{i i}^{\prime}=0 \\
\log \Psi_{i j}^{\prime}=1 / 2\left(\log \Psi_{i j}^{\prime}-\log \Psi_{j i}\right)
\end{gathered}
$$

then we get revised matrix $\left\|\log \Psi_{i j}^{\prime}\right\|$. The problem whether the ratio axiom (i) and (ii) are satisfied does not enter in this matrix. The raw mean scale and the column mean scale become identical;

$$
\begin{aligned}
\log \Psi_{i}^{\prime} & =-1 / m \sum_{j} \log \Psi_{i j}^{\prime} \\
& =1 / m \sum_{j} \log \Psi_{j i}^{\prime}
\end{aligned}
$$

which are equivalent to the $\log \hat{\psi}_{i}$ mentioned in the equation (26). We can recomend the scale $\Psi^{\prime}$ or $\hat{\psi}$ as a revised ratio scale of subjective heaviness.

However, we need further check about axiom (iii), by defining $\gamma_{i j}$ as follow

$$
\gamma_{i j}=\log \Psi_{i j}-\left(\log \Psi_{j}^{\prime}-\log \Psi_{i}^{\prime}\right) .
$$

When $\gamma$ is comparatively small, we can admit that the ratio axiom (iii) is satisfied by $\Psi_{i j}$.

Table 6 shows the obtained $\gamma$. They are not so large as $\delta$, but seems to have some systematic tendency. The absolute values in the cells near or far from the diagonal become large. That is, subjective ratios are likely to be over- or under-estimated when the paired stimuli are similar or dissimilar. Because of

$$
\sum_{j \gamma_{i j}}=0
$$

this bias does not affect the scale value $\Psi^{\prime}$. We obtain the matrix $\left\|\log \Psi_{i, j}^{*}\right\|$, by subtracting $\gamma$ from the matrix $\left\|\log \Psi_{i j}\right\|$, as shown in Table 7, which does not contain any constant error components.

\section{SUMmary}

(1) Categorical scales constructed by indirect methods satisfy Fechnerian log- arithmic law, while ratio scales constructed by direct methods satisfy Stevens' power law. Relationships between the two kinds of the scales have been discussed in terms of the criteria which were proposed by Stevens for differentiating prothetic and metathetic continua. We propose that in order to check validity of the scales the criteria should be revised and resolved into the two problems; the one concerns with definition of distribution function of sensation magnitudes and the other is about axioms for the level of measurements.

(2) Subjective magnitudes of weights, ranging from 40 to $200 \mathrm{~g}$, were measured of two direct methods; magnitude estimation method (Exp. I) and percentile method (Exp. II). The scale value increases in accordance with Stevens' power function. Logarithmically transformed values of the magnitudes show normal distributions and their variances are approximately constant.

The fact that direct estimations of the sensation magnitudes $\Psi$ show logarithmic normal distributions is not to be taken to expel the Fechnerian or Thurstonian idea that sensations $R$ obtained by indirect methods distribute by the normal. The difference between $R$ and $\Psi$ is the difference of their tastes about the definition of psychological values concerning the distribution function of the sensations. Curvilinear relationship of category scale and ratio scale may be artifact due to the difference of the sensation.

(3) We have found three kinds of deviations from the ratio axioms. They are the so-called constant errors (systematic errors) which are possible to be eliminated. It is not that only categorical methods produce constant errors and that time order errors and hysteresis are reducible to " category effect". Unless these biases are calculated and eliminated from the data of direct methods, we cannot admit them as the measurements of ratio scale. After the elimination, a revised ratio scale was recommended which is fairly agreeable 
to the veg scale.

\section{ReFERENCES}

EISLER, H. 1963 How prothetic is the continuun of smell? Scand. J. Psychol., 4, 29-32.

Goude, G. 1962 On fundamental measurement in psychology. Stochholm: Almquvist \& Wiksell.

Gulliksen, H. 1961 Linear and multidimensional scaling. Psychometrika, 26, 9-25.

Luce, R. D., \& Galanter, E. 1963 Discrimination ; psychological scaling. In Luce et. al. (Eds.) Handbook of mathematical psychology, I. New York: Wiley.

NAKAtani, K. 1965 Psychological scaling of sen- sation. Jap. J. Psychol., 36, 202-211.

Stevens, S.S. 1951 Mathematics, measurement and psychophysics. In Stevens (Ed.) Handbook of experimental psychology. New York: Wiley.

Stevens, S. S. 1957 On the psychophysical law. Psychol. Rev., 64, 153-181.

Stevens, S. S., \& Volkman, J. 1940 The relation of pitch to frequency: A revised scale. Amer. J. Psychol., 53, 329-353.

Tanaka, Y. 1961 Methods of psychological measurements. Tokyo: Univ. 'Tokyo Press.

Tanaka, Y. \& Nakatanr, K. 1967 On categorical scales of weight. Percept. Mot. Skills, 24, 143-150.

(Received July 14, 1967) 